\title{
Analysis of modifiable and nonmodifiable risk factors in patients undergoing pituitary surgery
}

\author{
Shane Shahrestani, MS, Alexander M. Ballatori, BA, Xiao T. Chen, BA, Andy Ton, BS, \\ Ben A. Strickland, MD, Andrew Brunswick, MD, and Gabriel Zada, MD, MS \\ Department of Neurosurgery, Keck School of Medicine, University of Southern California, Los Angeles, California
}

\begin{abstract}
OBJECTIVE Pituitary adenomas (PAs) are among the most common intracranial tumors. Understanding the clinical effects of various modifiable risk factors (MRFs) and nonmodifiable risk factors (NMRFs) is important in guiding proper treatment, yet there is limited evidence outlining the influence of MRFs and NMRFs on outcomes of PA resection. The aim of this study was to analyze MRFs and NMRFs in patients undergoing resection for PAs.
\end{abstract}

METHODS Using the 2016 and 2017 National Readmission Database, the authors identified a cohort of 9472 patients undergoing microscopic or endoscopic resection of a PA. Patients with nonoverlapping MRFs and NMRFs were analyzed for length of stay (LOS), hospital cost, readmission rates, and postoperative complications. From the original cohort, a subset of 373 frail patients (as defined by the Johns Hopkins Frailty Index) were identified and propensity matched to nonfrail patients. Statistical analysis included 1-way ANOVA, Tukey multiple comparisons of means, odds ratios, Wald testing, and unpaired Welch 2-sample t-tests to compare complications, outcomes, and costs between each cohort. Perioperative outcomes and hospital readmission rates were tracked, and predictive algorithms were developed to establish precise relationships between relevant risk factors and neurosurgical outcomes.

RESULTS Malnourished patients had significantly longer LOSs when compared to nonmalnourished patients $(p<0.001)$. There was a significant positive correlation between the number of MRFs and readmission at 90 days $(p=0.012)$ and 180 days $(p=0.020)$. Obese patients had higher rates of postoperative neurological injury at the 30 -day follow-up $(p=0.048)$ compared to patients with normal BMI. Within this NMRF cohort, frail patients were found to have significantly increased hospital LOS $(p<0.001)$ and total inpatient costs compared to nonfrail patients $(p<0.001)$. Predictive analytics showed that frail patients had significantly higher readmission rates at both 90 -day $(p<0.001)$ and 180 -day follow-ups $(p<0.001)$. Lastly, rates of acute postsurgical infection were higher in frail patients compared to nonfrail patients $(p<0.001)$.

CONCLUSIONS These findings suggest that both MRFs and NMRFs negatively affect the perioperative outcomes following PA resection. Notable risk factors including malnutrition, obesity, elevated lipid panels, and frailty make patients more prone to prolonged LOS, higher inpatient costs, and readmission. Further prospective research with longitudinal data is required to precisely pinpoint the effects of various risk factors on the outcomes of pituitary surgery.

https://thejns.org/doi/abs/10.3171/2020.4.JNS20417

KEYWORDS pituitary surgery; modifiable risk factors; predictive model; adenoma; sellar; frailty; BMI

$\mathrm{W}$ ITHIN the last several decades, the field of preventative medicine has grown immensely alongside an increased emphasis on avoiding unnecessary surgical care. ${ }^{1}$ A mainstay of preventive medicine includes thorough analysis of clinical effects of various modifiable risk factors (MRFs) and nonmodifiable risk factors (NMRFs). A wide range of MRFs and NMRFs, including BMI and age, have been associated with neurosurgical complications. ${ }^{2-4}$ Pituitary adenomas (PAs) are among the most common intracranial tumors; studies outlining the influence of MRFs and NMRFs on the outcomes of PA resection are limited, and none have used predictive analytics to evaluate the individual effects of MRFs and NMRFs on readmission rates and incidence of perioperative complications..$^{5}$ Although complication and readmission rates associated with resection of PAs are low overall, developing a holistic understanding of how these risk factors may influence outcomes is imperative in refining effectiveness of the procedure and guiding optimal case and patient selection. ${ }^{6,7}$ Doing so would allow physicians

ABBREVIATIONS GH = growth hormone; ICD-10 = International Classification of Diseases, Tenth Revision; JHACG = Johns Hopkins Adjusted Clinical Groups; LOS = length of stay; MRF = modifiable risk factor; NIS = National Inpatient Sample; NMRF = nonmodifiable risk factor; NRD = National Readmission Database; OR = odds ratio; $\mathrm{PA}=$ pituitary adenoma; SIADH = syndrome of inappropriate antidiuretic hormone secretion.

SUBMITTED February 10, 2020. ACCEPTED April 6, 2020.

INCLUDE WHEN CITING Published online June 12, 2020; DOI: 10.3171/2020.4.JNS20417. 
to more effectively alleviate the financial burden on this patient population, bearing in mind the high costs associated with the most common complications and prolonged length of stay (LOS). ${ }^{6}$ Furthermore, physicians would be better able to provide tailored patient counseling both preoperatively and postoperatively to align patient and family expectations.

As data continue to emerge regarding the role of MRFs and NMRFs in neurosurgical care, it is important to evaluate the influence of these factors as they pertain to PA resection. This investigation is the first study to leverage a large national database to study the effects of MRFs and NMRFs on PA resection and aims to clarify the precise relationships between relevant risk factors and perioperative outcomes in patients undergoing resection.

\section{Methods \\ Data Source}

We queried the National Readmission Database (NRD) from 2016 and 2017; the NRD is a yearly database provided by the Healthcare Cost and Utilization Project and the Agency for Healthcare Research and Quality. The NRD is a large, annual database that encompasses roughly half the US, with hospital readmission information for all ages, and more than 35,000,000 discharges in 2016 and 2017. These databases have been created to provide nationally representative information regarding inpatient hospital stays, readmissions, and their associated costs in the US. Diagnoses and procedures are coded within each patient admission or readmission as International Classification of Diseases, Tenth Revision (ICD-10) codes. NRD years 2015 and earlier were excluded due to their usage of ICD-9 coding, as they lack the granularity necessary for analysis.

\section{Patient Selection Criteria}

All inpatient hospitalizations involving microscopic or endoscopic PA resection were compiled to form a data set using ICD-10 codes. Demographics and hospital information were collected for each inpatient stay. Nonelective readmissions were analyzed in 30-day, 90-day, and 180-day intervals. Patients with MRFs including smoking (nicotine), alcoholism, elevated lipid panel, malnutrition (severe nutritional deficiencies, severe vitamin deficiencies, and starvation), hypertension, obesity (BMI $\geq 30 \mathrm{~kg} / \mathrm{m}^{2}$ ), and uncomplicated diabetes mellitus were identified using ICD-10 codes (Table 1). Frailty was the major NMRF included in the study, and frail patients were identified using ICD-10 codes and the Johns Hopkins Adjusted Clinical Groups (JHACG) frailty-defining diagnosis indicator. ${ }^{8-10}$ The JHACG frailty-defining diagnosis indicator uses a set of 10 clinical clusters (malnutrition, dementia, vision impairment, decubitus ulcer, urine control, weight loss, fecal control, social support, difficulty walking, and history of a fall) to predict patient frailty and has been validated through many studies with various patient populations. Propensity score matching was used to obtain a group of 373 nonfrail, one-to-one age- and sex-matched control patients from the initial lumbar spinal fusion cohort for comparison.
TABLE 1. ICD-10 codes for MRFs

\begin{tabular}{|c|c|}
\hline MRF & ICD-10 Code \\
\hline Smoking (nicotine) & $\begin{array}{l}\text { Z720, F17200, F17208, F17209, F17210, } \\
\text { F17213, F17218, F17219, F17220, F17223, } \\
\text { F17228, F17229, F17290, F17293, F17298, } \\
\text { F17299 }\end{array}$ \\
\hline Alcoholism & $\begin{array}{c}F 1010, F 10120, F 10121, F 10129, F 1014, F 10150, \\
\text { F10151, F10159, F10180, F10181, F10182, } \\
\text { F10188, F1019, F1020, F10220, F10221, } \\
\text { F10229, F10230, F10231, F10232, F10239, } \\
\text { F1024, F10250, F10251, F10259, F1026, } \\
\text { F1027, F10280, F10281, F10282, F10288, } \\
\text { F1029, F10920, F10921, F10929, F1094, } \\
\text { F10950, F10951, F10959, F1096, F1097, } \\
\text { F10980, F10981, F10982, F10988, F1099 }\end{array}$ \\
\hline Malnutrition & $\begin{array}{l}\text { E43, E440, E441, E46, E500, E501, E502, } \\
\text { E503, E504, E505, E506, E507, E508, E509, } \\
\text { E5111, E5112, E512, E518, E519, E52, E530, } \\
\text { E531, E538, E539, E534, E550, E559, E560, } \\
\text { E561, E568, E569, E58, E59, E60, E610, } \\
\text { E611, E612, E613, E614, E615, E616, E617, } \\
\text { E618, E619, E630, E631, E638, E639, E640, } \\
\text { E641, E642, E643, E648, E649, D530, D531, } \\
\text { D532, D538, D539 }\end{array}$ \\
\hline Elevated lipid panels & E7800, E782, E783, E781, E7841, E7849, E785 \\
\hline $\begin{array}{l}\text { Obesity (BMI } \geq 30 \\
\left.\mathrm{~kg} / \mathrm{m}^{2}\right)\end{array}$ & $\begin{array}{l}\text { Z6830, Z6831, Z6832, Z6833, Z6834, Z6835, } \\
\text { Z6836, Z6837, Z6838, Z6839, Z6841, Z6842, } \\
\text { Z6843 }\end{array}$ \\
\hline Hypertension & 110 \\
\hline $\begin{array}{l}\text { Uncomplicated } \\
\text { diabetes }\end{array}$ & $\begin{array}{l}\text { E100, E101, E106, E108, E109, E110, E111, } \\
\text { E116, E118, E119, E120, E121, E126, E128, } \\
\text { E129, E130, E131, E136, E138, E139, E140, } \\
\text { E141, E146, E148, E149 }\end{array}$ \\
\hline
\end{tabular}

\section{Statistical and Cost Analysis}

All statistical analysis was conducted in RStudio. Initial and readmission costs of pituitary adenoma resection were collected and compared between groups. Using ICD-10 codes, complications were collected for each readmission and were compared in both the MRF and NMRF groups. Statistical analysis methods included 1-way ANOVA, Tukey multiple comparisons of means, odds ratios (ORs), Wald testing, and unpaired Welch 2-sample t-tests to compare complications, outcomes, and costs between each cohort. ORs were visualized as a forest plot to highlight the influence of MRFs and NMRFs on patient readmission.

\section{Predictive Model Creation}

All predictive algorithms were developed using generalized gaussian linear regression models. Wald testing was performed to evaluate the effect of the weighted distance between the estimated value and the hypothesized true value under the null hypothesis on statistical parameters within each model. The predictive models were also graphically represented as dose-response curves, highlighting the effects of various risk factors on readmission. 


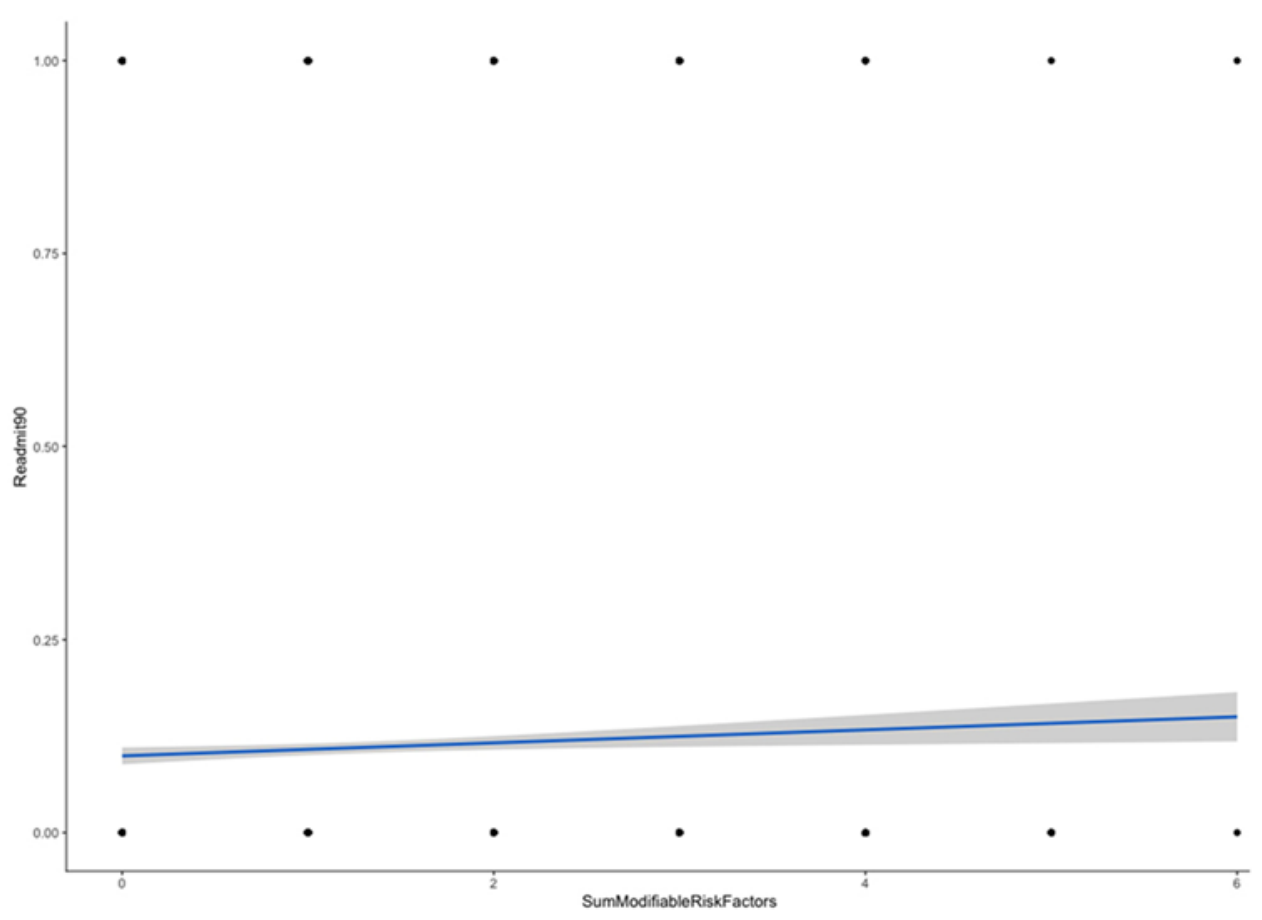

FIG. 1. Predictive linear regression model demonstrating an increase in readmission rates (blue line) associated with a greater number of MRFs at the 90 -day follow-up. Shaded areas represent the $95 \% \mathrm{Cls}$. Figure is available in color online only.

\section{Results \\ MRFs}

\section{Demographics}

In total, 9472 patients who underwent resection of a benign PA in 2016 or 2017 were identified. The mean patient age was $54.0 \pm 16.0$ years, with the sexes being equally represented (male 49.9\%, female 50.1\%). Microscopic resection was performed in 3792 cases (40.0\%) and endoscopic resection was performed in 5680 cases $(60.0 \%)$. Of these patients, $649(6.85 \%)$ had a growth hormone (GH)-producing adenoma, $405(4.28 \%)$ had a cortisolproducing adenoma, and $373(3.94 \%)$ had a prolactinoma. For the primary hospital admission, the average inpatient LOS was $4.53 \pm 6.89$ days and the total average cost of stay was $\$ 94,551 \pm \$ 103,036.80$. Malnourished patients had a significantly increased inpatient LOS $(8.78 \pm 11.74$ days) and total cost of primary admission $(\$ 163,002.20$ $\pm \$ 231,595.00)$ compared to patients with no MRFs ( $p<$ $0.001)$, elevated lipid panels ( $\mathrm{p}<0.001)$, hypertension ( $\mathrm{p}<$ $0.001)$, obesity ( $<<0.001)$, diabetes $(\mathrm{p}<0.001)$, and current nicotine use $(\mathrm{p}<0.001)$.

\section{Predictive Models}

At the 30-day follow-up, no statistical difference was identified between readmission rates and the number of risk factors in each individual. However, readmission at 90 days was significantly more likely with each increase in the number of risk factors ( $p=0.012$; Fig. 1). Predictive analytics found that each additional risk factor present contributed an additional $0.84 \%$ risk of readmission within 90 days. The same analysis at 180 days also found a positive correlation between 180-day readmission rates and the number of risk factors in each individual $(\mathrm{p}=$ 0.020; Fig. 2). Here, predictive analytics found that each additional risk factor contributed an additional $1.02 \%$ risk of being readmitted in 180 days.

\section{Complications and Readmissions}

The 30-, 90-, and 180-day readmission rates were found to be $7.96 \%, 11.00 \%$, and $13.15 \%$, respectively. No significant difference in readmission rates was found between patients with different MRFs at any follow-up intervals. Furthermore, no significant differences in infection, dural tears, CSF leak, pneumonia, syndrome of inappropriate antidiuretic hormone secretion (SIADH), and postoperative pain were found between patients with different MRFs at any follow-up intervals. However, obese patients had significantly higher rates of postoperative neurological complications at 30-day readmission compared to patients with no MRFs $(\mathrm{p}=0.048)$, but this finding was not seen at 90- and 180-day readmissions. Notably, patients with elevated lipid panels (OR 1.22, 95\% CI 1.04-1.42; p $=0.014)$ and malnutrition (OR 1.82, 95\% CI 1.29-2.50; $\mathrm{p}$ $<0.001)$ had significantly increased odds of readmission within 90 days (Figs. 3 and 4).

\section{NMRFs}

\section{Demographics}

Within the NMRF cohort, 373 frail patients and 373 one-to-one age- and sex-matched nonfrail patients were identified for analysis. The cohort containing frail patients had an average age of 63.7 years and comprised $41.6 \%$ fe- 


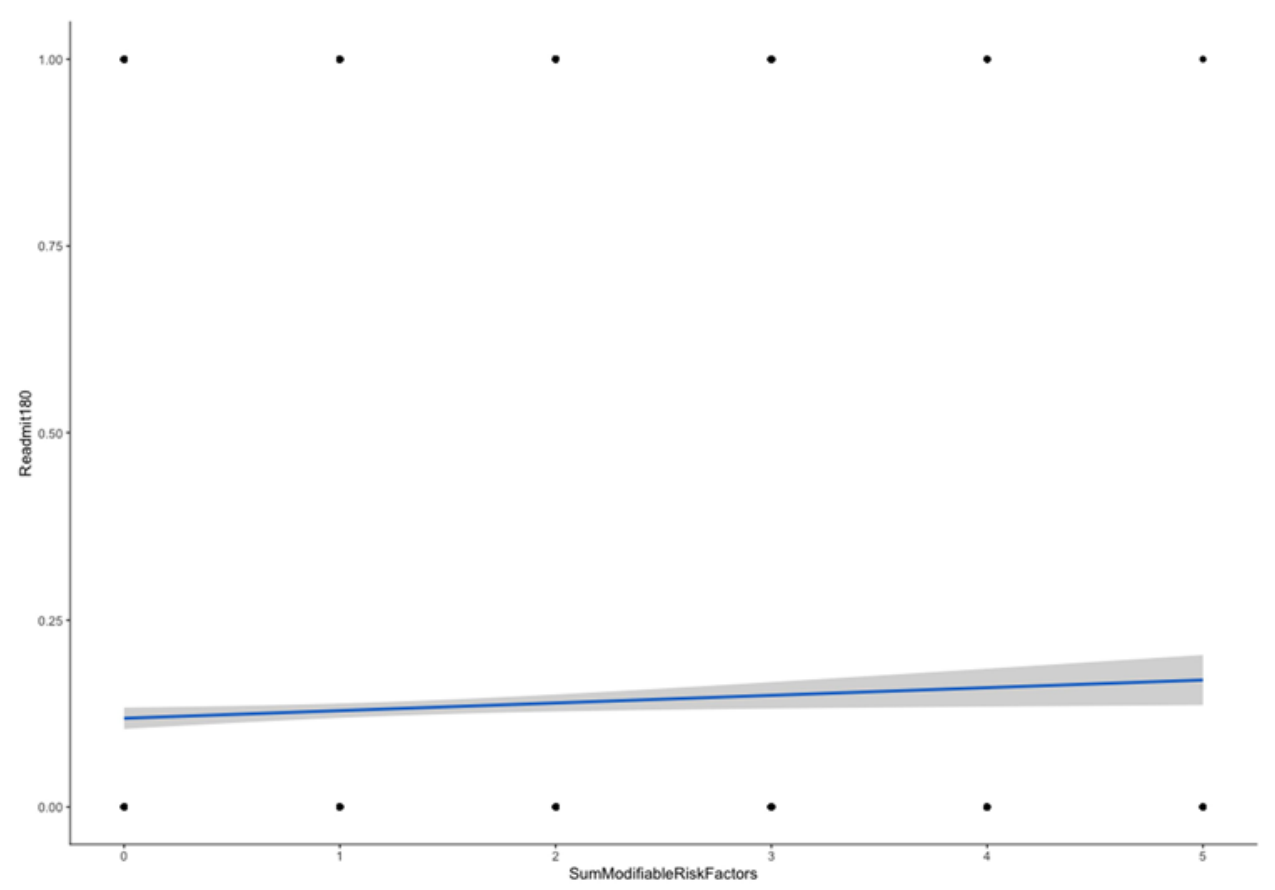

FIG. 2. Predictive linear regression model demonstrating an increase in readmission rates (blue line) associated with a greater number of MRFs at the 180 -day follow-up. Shaded areas represent the $95 \% \mathrm{Cls}$. Figure is available in color online only.

males, and the propensity-matched nonfrail cohort had an average age of 63.5 years and comprised $38.3 \%$ females. Microscopic resection was performed in 176 cases $(47.2 \%)$ and endoscopic resection was performed in 197 cases (52.8\%). Of these patients, 7 (1.88\%) had a GH-producing adenoma, $9(2.41 \%)$ had a cortisol-producing adenoma, and $12(3.22 \%)$ had a prolactinoma. A significant difference in LOS was seen between the frail (13.79 \pm 19.10 days) and nonfrail patients $(4.37 \pm 5.22$ days, $\mathrm{p}<0.001)$. The total inpatient hospital cost of frail patients $(\$ 191,129.27 \pm$ $\$ 244,619.10$ ) was significantly greater than that of nonfrail patients $(\$ 89,269.91 \pm \$ 82,787.67 ; \mathrm{p}<0.001)$.

\section{Predictive Models}

At the 30-day follow-up, no statistical difference was found between readmission rates and patient frailty. However, significant differences were found in patients at the 90- and 180-day follow-up. At 90 days, frail patients were found to have a significantly higher readmission rate $(\mathrm{p}<$ 0.001; Fig. 5). Predictive analytics found that frail patients had an additional $7.10 \%$ risk of readmission within 90 days. The same analysis at 180 days showed a positive correlation between 180-day readmission rates and the number of risk factors in each individual ( $p<0.001$; Fig. 6). Here, predictive analytics found that frail patients had an additional $11.28 \%$ risk of being readmitted within 180 days.

\section{Complications and Readmissions}

Frail patients had significantly higher 90-day (frail $17.80 \%$, nonfrail $10.71 \%, \mathrm{p}=0.0020$ ) and 180 -day (frail $23.98 \%$, nonfrail $12.70 \%, \mathrm{p}<0.001)$ readmission rates compared with nonfrail patients. However, no difference was seen at the 30-day follow-up (frail 8.93\%, nonfrail $7.92 \%, \mathrm{p}=0.51)$. Frail patients were also found to have higher rates of overall postsurgical infections during the primary admission (frail $6.43 \%$, nonfrail $0.00 \%$, p < 0.001 ). No significant differences were seen between the rates of CSF leak, dural tear, SIADH, and acute postsurgical anemia in frail and nonfrail patients.

\section{Discussion}

Our findings suggest that malnourished patients have significantly higher LOS when compared to all other patients. The number of MRFs present in patients was associated with significantly increased risk of readmission within 90 and 180 days, and obese patients were found to have higher rates of postoperative complications at the 30-day follow-up. Predictive analytics showed that frail patients had significantly higher readmission rates at both 90- and 180-day follow-ups, and rates of acute postsurgical infections were higher in frail patients when compared to nonfrail patients undergoing PA resection.

Although the literature outlining the independent effects of various risk factors on the outcomes of PA resection remains limited, previous studies have examined relationships between several risk factors and postsurgical complications. Although some studies found a correlation between increased BMI $(>30)$ and increased rates of postoperative CSF leakage, our study and others have found no correlation. ${ }^{11-15}$ The likely reason for this discrepancy is twofold: first, all literature correlating BMI to CSF leakage utilizes smaller sample sizes than that of our cohort and thus is less powered; and second, the published litera- 
Shahrestani et al.

$\begin{array}{lrrr}\text { Modifiable Factors } & \text { Readmitted } & \text { Not Readmitted } & \text { OR } \\ \text { Smoking } & 71 & 567 & 1.02 \\ \text { Alcohol Use } & 8 & 51 & 1.29 \\ \text { Elevated Lipid Panel } & 272 & 1924 & 1.22 \\ \text { Malnutrition } & 46 & 211 & 1.82 \\ \text { Hypertension } & 386 & 2925 & 1.13 \\ \text { Obesity } & 158 & 1284 & 1.00 \\ \text { Diabetes - Uncomplicated } & 113 & 897 & 1.02\end{array}$

One or More MRF

1.33

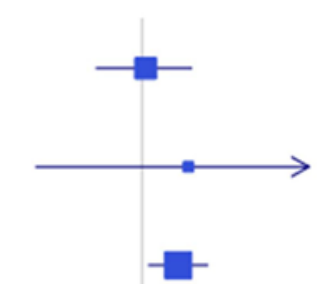

FIG. 3. Forest plots showing ORs of MRFs at the 90-day follow-up. Figure is available in color online only.

$\begin{array}{lrrr}\text { Modifiable Factors } & \text { Readmitted } & \text { Not Readmitted } & \text { OR } \\ \text { Smoking } & 66 & 385 & 1.15 \\ \text { Alcohol Use } & 5 & 30 & 1.13 \\ \text { Elevated Lipid Panel } & 219 & 1300 & 1.17 \\ \text { Malnutrition } & 29 & 140 & 1.39 \\ \text { Hypertension } & 318 & 1970 & 1.13 \\ \text { Obesity } & 133 & 860 & 1.03 \\ \text { Diabetes - Uncomplicated } & 101 & 604 & 1.12\end{array}$

One or More MRF

1.44
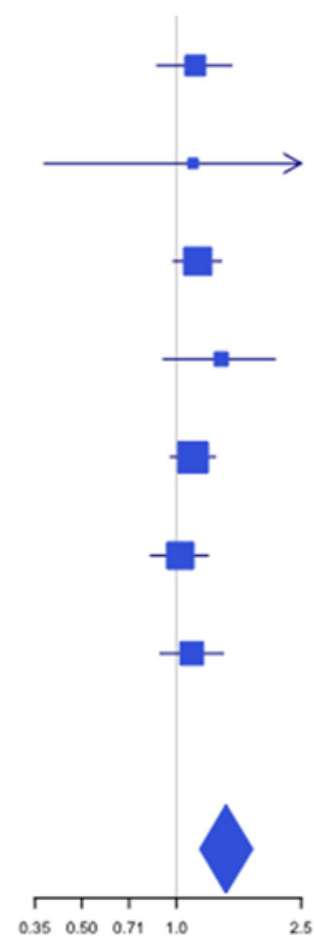

FIG. 4. Forest plots showing ORs of MRFs at the 180-day follow-up. Figure is available in color online only. 


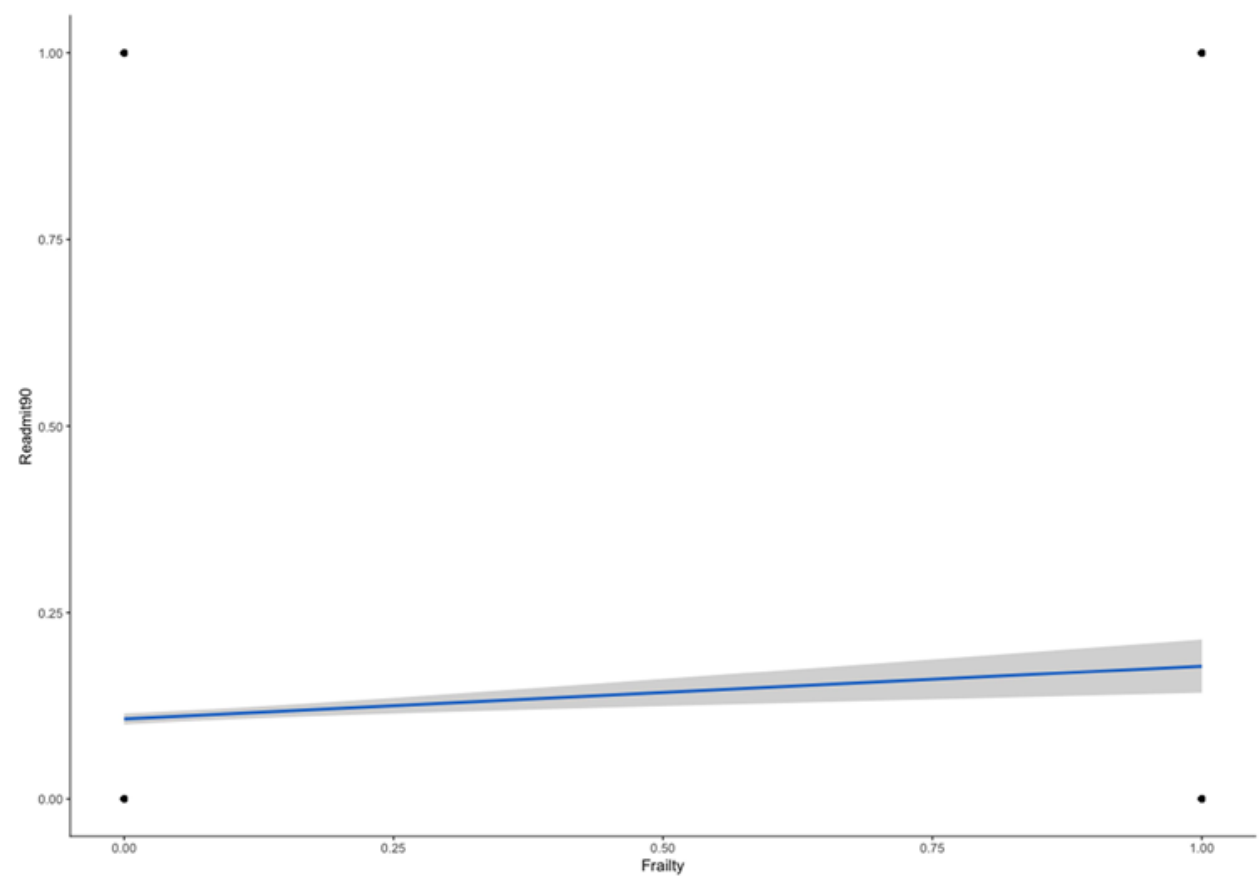

FIG. 5. Predictive linear regression model demonstrating increased readmission (blue line) associated with a diagnosis of frailty at the 90 -day follow-up. Shaded areas represent the $95 \%$ Cls. Figure is available in color online only.

ture focuses exclusively on the correlation between BMI and CSF leak in endoscopic cohorts, while our analysis is performed on a cohort containing both microscopic and endoscopic procedures. Although no correlation was found between BMI and CSF leakage, there was a significant association between increased BMI and neurological injury at 30-day readmission, but this association was not found at 90 - and 180-day follow-up. This finding suggests that

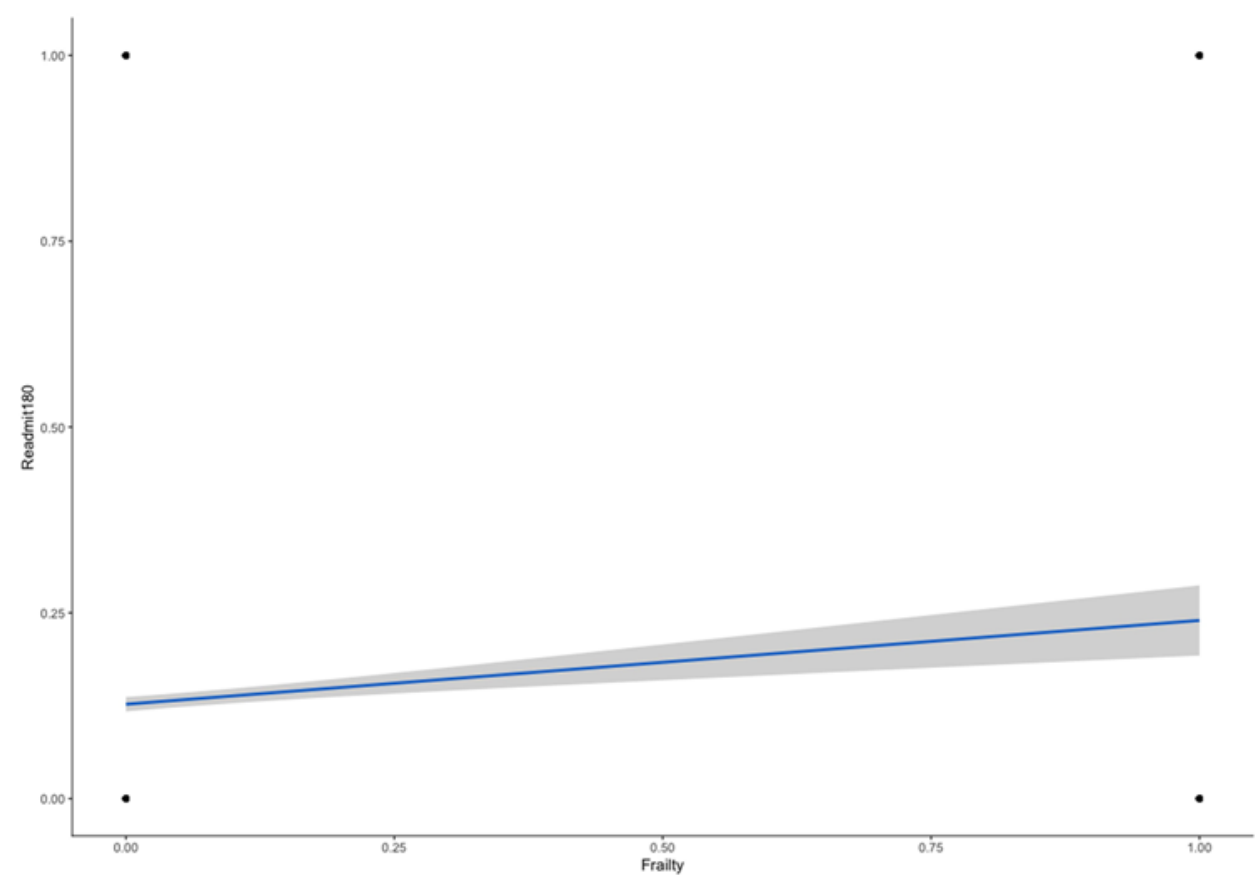

FIG. 6. Predictive linear regression model demonstrating increased readmission (blue line) associated with a diagnosis of frailty at the 180 -day follow-up. Shaded areas represent the $95 \% \mathrm{Cls}$. Figure is available in color online only. 
rates of postsurgical neurological injury may start off as significant for patients with different BMIs and then level out as follow-up time increases past 30 days. Furthermore, several studies outlining the influence of modifiable risk factors on postoperative rates of intracranial infection have arrived at mixed conclusions, with some studies claiming no significance and others finding a small increase in infection rates in diabetic patients..$^{11,16,17}$ In the current study, no significant postoperative associations between diabetes mellitus and infection rates were seen.

The Johns Hopkins Frailty Index is a validated metric that allows clinicians and researchers to evaluate and compare patients' health status. ${ }^{8-10}$ This index has previously been used to assess frailty's impact on short-term outcomes of patients undergoing pituitary surgery. Asemota and Gallia conducted a propensity-matched study of frail versus nonfrail patients undergoing transsphenoidal pituitary surgery using the 2000-2014 National Inpatient Sample (NIS), and found that frail patients had significantly higher rates of fluid and electrolyte disorders, intracranial vascular complications, mental status changes, pulmonary insufficiency, acute kidney failure, and other postoperative complications. ${ }^{18}$ Our study corroborates these findings, as our propensity-matched analysis of frail and nonfrail patients showed that frailty was significantly associated with higher rates of nonelective readmissions at 90 and 180 days. In addition, both the prior NIS and current NRD studies found that frail patients had significantly higher mean total charges and longer hospitalizations. ${ }^{18}$ Both of these analyses of PA resection agree with the current understanding of frailty in neurological surgery, as a recent literature review of 25 studies that focused on the heterogeneity of frailty in neurosurgery found that frailty increased complication rates, mortality rates, and LOS..$^{19}$

The use of predictive models and forest plots in this study allowed for data visualization and the creation of dose-response curves correlating MRFs and NMRFs to increased readmission rates and complications. Previous studies have demonstrated the efficacy of predictive models in forecasting early readmissions in pituitary surgery. ${ }^{20}$ Notably, the study conducted by Hollon et al. ${ }^{20}$ found that obesity was a good predictor for postsurgical complications, and we found similar results correlating obesity and neurological complications at the 30-day follow-up. The use of forest plots also allowed for significant ORs to be visualized and highlighted an increased risk of readmission at 90 and 180 days in patients diagnosed with malnutrition and elevated lipid panels.

\section{Limitations}

This study has several limitations. First, this study is limited by its retrospective analysis during a narrow range of time (2016 and 2017 only). However, the dates were chosen due to the implementation of mandatory ICD10 coding in late 2015, which allowed for more detailed codes to be drawn for analysis. Despite these advances in coding, overlapping codes prevented thorough subgroup analysis (e.g., the code for Cushing's disease encapsulated Cushing's-related weight gain, leading to discrepancies in BMI coding). Thus, despite the limited time window and decreased potential sample size, this study was able to achieve greater granularity than any previous studies that used ICD-9 codes. Furthermore, as a retrospective cohort study, data were limited by the quality and depth of the records kept for all patients. Another study limitation is that the NRD encapsulates roughly $60 \%$ of the US population, so the conclusions drawn in this study may not be widely representative of the entire US population. Using a national, de-identified database, we also lack patient-reported outcomes of pain and disability following each surgical procedure as these outcomes lack specific ICD-10 codes, and the data are prone to coding errors and discrepancies in coding between hospitals. This study also did not analyze differences in elective readmissions between groups. Lastly, while several frailty measures are available, the criteria for some are not included in the NRD. This is why we used only the JHACG frailty-defining diagnosis indicator, which includes the necessary ICD-10 codes to accurately define frailty in our patient cohort.

\section{Conclusions}

Overall, our findings suggest that both modifiable and nonmodifiable risk factors negatively affect perioperative outcomes in patients undergoing PA resection. Notable risk factors including malnutrition, obesity, elevated lipid panels, and patient frailty make patients more prone to prolonged hospital stay, higher associated inpatient costs, and complications resulting in readmission. Further research with prospective multicenter longitudinal data is required to precisely pinpoint the effects of various risk factors on the outcomes of pituitary surgery.

\section{References}

1. Pomerleau O, Bass F, Crown V. Role of behavior modification in preventive medicine. N Engl J Med. 1975;292(24):12771282.

2. Garavaglia M, Mak T, Cusimano MD, et al. Body mass index as a risk factor for increased serum lactate during craniotomy. Minerva Anestesiol. 2013;79(10):1132-1139.

3. Oh T, Safaee M, Sun MZ, et al. Surgical risk factors for postoperative pneumonia following meningioma resection. Clin Neurol Neurosurg. 2014;118:76-79.

4. Qureshi AI, Suarez JI, Parekh PD, et al. Risk factors for multiple intracranial aneurysms. Neurosurgery. 1998;43(1):22-27.

5. Molitch ME. Diagnosis and treatment of pituitary adenomas: a review. JAMA. 2017;317(5):516-524.

6. Hendricks BL, Shikary TA, Zimmer LA. Causes for 30-day readmission following transsphenoidal surgery. Otolaryngol Head Neck Surg. 2016;154(2):359-365.

7. Agam MS, Wedemeyer MA, Wrobel B, et al. Complications associated with microscopic and endoscopic transsphenoidal pituitary surgery: experience of 1153 consecutive cases treated at a single tertiary care pituitary center. J Neurosurg. 2019;130:1576-1583.

8. Lieberman R, Abrams C, Weiner JP. Development and Evaluation of the Johns Hopkins University Risk Adjustment Models for Medicare+Choice Plan Payment. Johns Hopkins University Press; June 6, 2003. Accessed April 22, 2020. https://www.hopkinsacg.org/document/development-andevaluation-of-the-johns-hopkins-university-risk-adjustmentmodels-for-medicarechoice-plan-payment/

9. The Johns Hopkins ACG Technical Reference Guide, version 9.0. Johns Hopkins University; 2009. Accessed April 22, 2020. https://www.hopkinsacg.org/document/acg-systemversion-9-0-full-documentation/ 
10. Sternberg SA, Bentur N, Abrams C, et al. Identifying frail older people using predictive modeling. Am J Manag Care. 2012;18(10):e392-e397.

11. Boling CC, Karnezis TT, Baker AB, et al. Multi-institutional study of risk factors for perioperative morbidity following transnasal endoscopic pituitary adenoma surgery. Int Forum Allergy Rhinol. 2016;6(1):101-107.

12. Dlouhy BJ, Madhavan K, Clinger JD, et al. Elevated body mass index and risk of postoperative CSF leak following transsphenoidal surgery. J Neurosurg. 2012;116(6):1311-1317.

13. Senior BA, Ebert CS, Bednarski KK, et al. Minimally invasive pituitary surgery. Laryngoscope. 2008;118(10):18421855.

14. Karnezis TT, Baker AB, Soler ZM, et al. Factors impacting cerebrospinal fluid leak rates in endoscopic sellar surgery. Int Forum Allergy Rhinol. 2016;6(11):1117-1125.

15. Lee YJ, Wong A, Filimonov A, et al. Impact of body mass index on perioperative outcomes of endoscopic pituitary surgery. Am J Rhinol Allergy. 2018;32(5):404-411.

16. Lobatto DJ, de Vries F, Zamanipoor Najafabadi AH, et al. Preoperative risk factors for postoperative complications in endoscopic pituitary surgery: a systematic review. Pituitary. 2018;21(1):84-97.

17. Zhang L, Chen M. Analysis of factors causing intracranial infection after endoscopic resection of pituitary tumors by transnasal-sphenoidal approach. Biomed Res. 2014;25(4):437440.

18. Asemota AO, Gallia GL. Impact of frailty on short-term outcomes in patients undergoing transsphenoidal pituitary surgery. J Neurosurg. 2019;132(2):360-370.
19. Pazniokas J, Gandhi C, Theriault B, et al. The immense heterogeneity of frailty in neurosurgery: a systematic literature review. Neurosurg Rev. Published online January 17, 2020. doi:10.1007/s10143-020-01241-2

20. Hollon TC, Parikh A, Pandian B, et al. A machine learning approach to predict early outcomes after pituitary adenoma surgery. Neurosurg Focus. 2018;45(5):E8.

\section{Disclosures}

The authors report no conflict of interest concerning the materials or methods used in this study or the findings specified in this paper.

\section{Author Contributions}

Conception and design: Shahrestani. Acquisition of data: Shahrestani. Analysis and interpretation of data: Shahrestani. Drafting the article: Shahrestani, Ballatori, Chen, Ton. Critically revising the article: Ballatori, Chen, Ton. Reviewed submitted version of manuscript: Strickland, Brunswick, Zada. Statistical analysis: Shahrestani. Administrative/technical/material support: Strickland, Brunswick, Zada. Study supervision: Strickland, Brunswick, Zada.

\section{Correspondence}

Shane Shahrestani: Keck School of Medicine, University of Southern California, Los Angeles, CA.shanesha@usc.edu. 